\title{
INVESTIGACIÓN MICROEPIDEMIOLÓGICA DE STRONGYLOIDES STERCORALISEN AMBIENTES DOMICILIARIOS DE PACIENTES INFECTADOS CON EL VIRUS DE LA INMUNODEFICIENCIA HUMANA (VIH)
}

\section{MICROEPIDEMIOLOGICAL INVESTIGATION OF STRONGYLOIDES STERCORALIS IN INFECTED PATIENTS HOUSES WITH THE HUMAN IMMUNODEFICIENCY VIRUS (HIV)}

Fatima Helena Cecchetto', Marco Túlio Antonio García-Zapata², Jose Luis Barros Araújo², Edson Sidião Souza Jr. ${ }^{2}$, Marcia Livia MarQues Faria ${ }^{2}$, Fernanda Barbosa de Oliveira ${ }^{2} \&$ Gustavo Barbosa de Oliveira ${ }^{2}$

Fecha de recepción: 09 de febrero de 2007, fecha de aceptación: 27 de marzo de 2007.

Forma de citar: Ceccheto, FH, García-Zapata, MTA, Araújo, BJL, Souza, ESIr, Faria MM, Oliveira FB \& Oliveira GB. 2007. Investigación microepidemiológica de Strongyloides stercoralis en ambientes domiciliarios de pacientes infectados con el Virus de la Inmunodeficiencia Humana. Neotropical Helminthology, vol. 1, no. 1, pp. 31-36.

\section{Resumen}

Existe una posible asociación entre la infección por parásitos intestinales de pacientes inmunosuprimidos y la contaminación de su ambiente domiciliar. Por eso, esta asociación fue estudiado a través de un estudio microepidemiológico para determinar la presencia de helmintos y coccidios intestinales en muestras obtenidas de suelo, agua y verduras de uso común y constante en las viviendas de los pacientes inmunosuprimidos $(\mathrm{VIH}+$, linfocitos CD4 $\geq 200 \mathrm{~mL}$, carga viral $\approx 10$ mil). Las muestras fueron seleccionadas por muestreo aleatorio y sistematizado. Para el análisis del suelo fueron utilizadas las técnicas de Cadwell-Cadwell, Hoffmann, Pons \& laner (HPI) y Kinyoun modificado (caliente) mientras Que para las muestras de agua y verdura fueron usadas HPI, Faust, y la técnica de filtración con filtro de propil-etileno. Nosotros encontramos larvas rabditiformes de Strongyloides stercoralis en Allium fistolosum (cebollita china) cultivado en la huerta de la residencia de uno de los 1 I pacientes evaluados. Esta forma parasitaria a pesar de no ser infectante para el ser humano, es parte del ciclo biológico de $S$. stercoralis y es fundamental para su multiplicación y manutención en el medio ambiente. Este hallazgo denota la importancia ecológica del ser humano en la dinámica de la transmisión de las geohelmintiasis, y alerta la necesidad de instruir a la población sobre medidas profilácticas e higiene, especialmente, de pacientes inmunosuprimidos.

Palabras clave: Strongyloides stercoralis - Helmintos - Allium fistolosum - Síndrome de Inmunodeficiencia AdQuirida - Inmunosupresión - Contaminación Ambiental.

\section{Abstract}

A possible association between intestinal parasite infection in immunocompromised patients with and the contamination their domiciliary environment may exist. Therefore, this correlation was studied through a study microepidemiologic by determining the presence of helminths and intestinal coccidian in samples obtaneid from the floor, water and vegetables of common and constant use in houses of patients with the Human Immunodeficiency Virus (HIV+, CD4 lymphocytes $\geq 200 \mathrm{~mL}$, viral load $\approx 10$ thousand). The samples were selected by random and systematized sampling. For the analysis of the floor the techniques of Cadwell-Cadwell, Hoffmann, Pons \& Janer (HPI) and modified Kinyoun (hot) were used whereas HPI, Faust, and the filtration technique with propilethylene filter were used for water and vegetables samples. We found rhabditiform larves of Strongyloides stercoralis in Allium fistolosum (Chinese onion) cultivated in the vegetable garden of one of the II patient's. Although this parasitic form is a non-infective stage for the human, it makes part of the biological cycle of $S$. stercoralis and it is fundamental for its multiplication and maintenance in the environment. This finding denotes the human ecological importance in the dynamics of the transmission of geohelminths, and alerts to necessity of instructing the population on measured prophylaxes and hygiene, especially to immunocompromised patients.

Keywords: Strongyloides stercoralis - Helminths - Allium fistolosum - Aceuired Immunodeficiency Syndrome - Inmunossuppression - Environmental Pollution.

I. Faculdade Nossa Senhora de Fátima, Caxias do Sul - RS, Brasil

2. Instituto de Patologia Tropical e Saúde Pública (IPTSP) / Universidade Federal de Goiás, Goiânia - GO, Brasil 


\section{INTRODUCCIÓN}

Las parasitosis intestinales por su elevada diversidad de manifestaciones clínicas, representan un problema de gran importancia en salud pública, principalmente en países donde, aun, son insatisfactorias las condiciones de saneamiento básico. Estas son una de las principales causas debilitantes de una población, y frecuentemente están asociadas a cuadros de diarrea y desnutrición, comprometiendo el desarrollo físico e intelectual de la población (Oliveira \& Germano, 1992; Silva et al., 1995).

Entre los factores Que contribuyen para la diseminación de enteroparásitos, merece destacar la contaminación fecal del suelo y del agua. Todas las condiciones oue favorezcan esta situación deben ser investigadas con detenimiento (Gassi et al., 1984; Oliveira, 1991; Guilherme et al., 1999; Mesquita et al., 1999).

En el caso de Strongyloides stercoralis (Bavay, 1876), la forma Que representa riesgo de contaminación para el hombre es solamente la larva filariforme. Las larvas rabditiformes son eliminadas en las heces, contaminan el medio ambiente, y toman dos caminos, o se transforman directamente en larvas infectantes filariformes, o hacen un camino intermediario transformándose en machos $y$ hembras de vida libre, cuyos huevos posteriormente liberarán larvas rabdtiformes y se transformarán en filariformes, contaminando también el medio ambiente. Este hecho sugiere que la defecación en el campo de individuos infectados $\mathrm{y} / \mathrm{o}$ con hábitos higiénicos precarios se constituyen en un elevado riesgo para la contaminación de pacientes susceptibles que no utilizan calzados o manipulan tierra contaminada, pudiendo originar cuadros clínicos locales (cutáneos, o subcutáneos), como "larva migrans" o "larva currens" (en la región peri-anal), o de mayor gravedad Ipulmonares, intestinales y diseminados] (Rey, 200I).

Este estudio fue realizado con el objetivo de evaluar la posible asociación entre la infección de pacientes inmunosuprimidos con parásitos entéricos y la contaminación del medio ambiente domiciliar Que habitan. Pretende, de forma general, dimensionar la importancia de la dinámica ecológica humana en la transmisión de las enteroparasitosis en este tipo especial de pacientes.

\section{MATERIAL Y MÉTODOS}

Este trabajo es parte de un estudio longitudinal sobre agentes oportunistas infecciosos en pacientes inmunosuprimidos, la mayoría con sida $(\mathrm{VIH}+$, linfocitos $\mathrm{CD} 4$ $\geq 200 \mathrm{~mL}$, carga viral $\approx 10$ mil) portadores de enteroparasitosis, siguieron el flujograma dinámico implantado para el estudio. Este trabajo contó con la aprobación del Comité de Ética de la Universidad Federal de Goiás, y para enrolar a los participantes utilizamos el consentimiento informado en todos los casos.

AQuí, será descrita la evaluación del medio ambiente domiciliar de un paciente infectado con un helminto intestinal, encontrado dentro de un grupo de once pacientes con sida portadores de otras parasitosis intestinales (principalmente protozoos), Que será motivo de un estudio separado.

Fue realizado en la ciudad de Goiania, estado de Goiás, localizado en plena meseta brasileña, a $180 \mathrm{~km}$ de la capital (Brasilia-DF), después del período lluvioso (Temperatura $=15-27^{\circ} \mathrm{C}$; humedad $=55-60 \%$ ). Cuando fueron investigadas de forma aleatoria y sistematizada muestras del suelo, agua y verduras de uso común y constante en las vivienda del paciente investigado.

Fueron colectadas cinco muestras del suelo ( 3 de la región peridomiciliar y 2 de la huerta), 4 litros de agua de consumo de la familia, tres muestras de verdura usadas en la preparación de alimentos de la familia, una muestra fue de tomate (Lycopersicun esculentum Mill) adQuirido en el mercado cerca de la vivienda, $y$ las otras 2 de "cebollita china" (Allium fistolusum $\mathrm{L}$ ) cultivado en la propia huerta de la residencia.

Las muestras del suelo fueron colocadas en frascos estériles, las verduras en bolsas plásticas, el agua en frascos de vidrio y plásticos, previamente lavados y esterilizados. Para el análisis del suelo fueron utilizadas las técnicas de Cadwell-Cadwell, Hoffmann, Pons \& laner (HPI), Faust, y coloreados con Kinyoun caliente para el diagnóstico, de helmintos y coccidios intestinales, respectivamente. (CETSB, 1989; De Carli, 2000 \& D'Agosto et al., 2000).

Las verduras fueron lavadas con detergente neutro $y$, con auxilio de un pincel, cuidadosamente refregadas, el lícuido obtenido fue filtrado y dejado en reposo durante 
24 h. Aquí, fueron utilizados los métodos HPJ, Faust y coloreados con Kinyoun caliente (Oliveira et al., 1992; Branco et al., 1998; Guilherme et al., 1999; Mesquita et al., 1999).

La técnica de filtración utilizada para el agua fue el de la membrana filtrante de etil-propiletileno - Millipore ${ }^{\circledR}$ (tipo H.A 0,45 $\mu \mathrm{m}$ ) adaptada para el estudio de coccidios (Rose et al., 1989; Gamba et al., 2000).

\section{RESULTADOS}

En el ambiente domiciliar de uno de los once pacientes infectados con el virus de la Inmunodeficiencia Humana (VIH) se evidenció la presencia de larvas rabditiformes de $S$. stercoralis contaminando el $A$. fistolusum ("cebollita china"), Que coincidió con la infección parasitaria del único paciente con sida que la habitaba. La residencia estaba constituida por dos cuartos, y estaba localizada en una región periférica de clase media-baja. Tenía características semirurales. Sin asfalto y sin condiciones mínimas de saneamiento básico (sin un baño y sin una red de agua y desagüe). La huerta de la casa (no tenía ninguna cerca y estaba situada en los fondos del terreno), era compartida con una familia vecina, y con frecuencia era utilizada por el propio paciente (por sus diarreas abundantes e incontrolables) para sus necesidades biológicas. No existía baño ni ninguna fosa negra, y el desagüe era eliminado a "cielo abierto". La colecta de basura, por el sistema público municipal, sucedía dos veces por semana. El agua utilizada para su consumo procedía de un pozo artesanal (no potable), y era apenas filtrada, a través de un dispositivo simple ("vela").

Los aspectos sanitarios de los otros 10 pacientes con VIH y sin parasitosis intestinal, pueden ser observados en el Tabla 1.

Tabla 1. Condiciones sanitarias de ambientes domiciliarios de pacientes infectados con el virus de inmunodeficiencia humana sin ninguna evidencia de parasitosis intestinal a través de técnicas coprológicas específicas, Goiania, Brasil.

\begin{tabular}{|c|c|c|c|c|}
\hline $\begin{array}{c}\text { Origen } \\
\text { del agua } \\
\text { consumida }\end{array}$ & $\begin{array}{l}\text { Tratamiento } \\
\text { del agua } \\
\text { antes de su } \\
\text { consumo }\end{array}$ & $\begin{array}{l}\text { Localización } \\
\text { de las } \\
\text { viviendas }\end{array}$ & $\begin{array}{l}\text { Destino del } \\
\text { desagüe } \\
\text { de la vivienda }\end{array}$ & $\begin{array}{l}\text { Destino } \\
\text { de la basura } \\
\text { conectada }\end{array}$ \\
\hline $\begin{array}{l}\text { Potabilización } \\
\text { municipal } \\
6 / 10\end{array}$ & $\begin{array}{l}\text { Hervida } \\
1 / 10\end{array}$ & $\begin{array}{l}\text { Urbana } \\
6 / 10\end{array}$ & $\begin{array}{l}\text { Servicio } \\
\text { Municipal } \\
2 / 10\end{array}$ & $\begin{array}{l}\text { Colecta } \\
\text { municipal } \\
9 / 10\end{array}$ \\
\hline \multirow[t]{2}{*}{$4 / 10$} & $\begin{array}{l}\text { Filtrada } \\
7 / 10\end{array}$ & $\begin{array}{l}\text { Semi-rural } \\
4 / 10\end{array}$ & $\begin{array}{l}\text { Baño } \\
7 / 10\end{array}$ & $\begin{array}{c}\text { Enterrado } \\
1 / 10\end{array}$ \\
\hline & $\begin{array}{c}\text { Ninguna } \\
2 / 10\end{array}$ & & $\begin{array}{c}\text { Cielo abierto } \\
1 / 10\end{array}$ & \\
\hline
\end{tabular}

\section{DISCUSIÓN}

Las enfermedades transmitidas por alimentos y por el medio ambiente son resultantes, predominantemente, del ciclo de contaminación fecal/oral. Actualmente, en el mundo como en el Brasil pocos son los trabajos que evalúan la dinámica de contaminación del medio ambiente sobre los pacientes inmunosuprimidos y viceversa.

El resultado obtenido en este estudio muestra la importancia del medio ambiente en la transmisión de los enteroparásitos, tipo $S$. stercoralis Que necesitan del suelo para completar su ciclo biológico y multiplicarse. Se debe resaltar en este estudio la asociación de la contaminación ambiental con la del propio paciente, Que en este caso especial es un inmunosuprimido. Debe considerarse también, Que en esa vivienda no existían condiciones sanitarias mínimas (ausencia de sanitario privado en el propio domicilio).

La larva rabditiforme encontrada en la "cebollita china", indispensable para la manutención del parásito en el ambiente, podría seguir dos ciclos evolutivos: el directo, transformándose en filariforme, forma infectante para el hombre, y el ciclo indirecto permaneciendo en el suelo, convirtiéndose en adultos de vida libre, macho o hembra, Que copulan, liberan sus huevos transformándose en larvas rabditiformes y luego en filariformes, pudiendo permanecer viables por semanas en el suelo, hasta alcan- 
zar un huésped susceptible y penetrar por piel y mucosas y continuar su desarrollo hasta llegar a constituirse como una hembra partenogenética (Pesssoa \& Martins, 1988).

En el ciclo indirecto las generaciones de vida libre pueden permanecer una o más generaciones en el medio ambiente, dependiendo de las condiciones climáticas (temperatura y humedad) donde se encuentran. Estudios recientes mencionan Que las larvas resisten a bajas temperaturas, tienen mayor porcentaje de supervivencia en climas subtropicales con lluvias bien distribuidas, con temperatura elevada y en suelos arenosos, como el correspondiente al área descrita aquí (Rey, 2001).

Resaltamos, también, Que en la literatura científica revisada no encontramos mención sobre el encuentro de parásitos en $A$. fistolosum. Sin embargo, hay diversos trabajos Que mencionan la presencia de Quistes de protozoarios y huevos de helmintos en diferentes tipos de hortalizas, tales como lechuga, berro y achicoria (Germano et al., 1991; Silva et al., 1995; Guilherme et al., 1999). Hay inclusive reportes Que encuentran, aproximadamente, 20\% de las hortalizas contaminadas por parásitos entéricos, de ellos 1-4\% son por $S$. stercoralis (Guilherme et al., 1999; Takayanagui et al., 2000).

Se sabe también, Que la principal forma de contaminación se efectúa: ( 1 ) a través del agua contaminada por material fecal, utilizada en irrigaciones; (2) por contaminación del suelo a través del uso de abono orgánico con excremento fecal; $y$, (3) por contacto con el agua y alimentos con las manos contaminadas (Ludwig et al., 1999).

Esos trabajos, y el nuestro, revelan Que a pesar Que cada tipo de parásito presenta ciclos biológicos diferentes, la mayoría de los monoxénicos tienen en común el hecho de que su diseminación ocurre a través de la contaminación ambiental por Quistes, huevos o larvas, eliminados junto con las heces de individuos y animales parasitados.

Por la dinámica del proceso y metodología aplicada, resulta difícil concluir cuál fue la verdadera situación de nuestro caso. Puesto que la contaminación del suelo donde era cultivada la "cebollita china" podría haber sido la fuente infectante para nuestro paciente, al ingerir esta verdura fresca (con larvas rabditiformes, Que maduraron para la forma infectante: larvas filariformes) sin seguir las mínimas recomendaciones de higiene, o lo contrario, Que el propio paciente sea el responsable de la contaminación del ambiente domiciliar donde era cultivada esta verdura. En cualquier caso, una de estas hipótesis muestra la evidencia Que la verdura contaminada constituye un riesgo importante para todos los habitantes de esa vivienda, Que de alguna manera, se exponen al contacto con el suelo contaminado, y así pueden adeuirir la estrongyloidiosis.

En el caso específico de nuestro paciente con cuadro diarreico, Que frecuentaba ese suelo contaminado, ese hecho podría significar continuas y repetidas infecciones por este tipo de agente, agravando su cuadro clínico ya deteriorado por la inmunodeficiencia resultado de la enfermedad de fondo (sida) independiente de las posibles auto-infestaciones internas y/o diseminación del S. stercoralis, Que con frecuencia suceden en pacientes inmunosuprimidos (especialmente los sometidos a corticoterapia prolongada).

La ausencia de saneamiento básico y de sanitarios en el ambiente domiciliar refuerza la posibilidad de la existencia de un ciclo de transmisión vicioso en el local, donde el hombre infectado contamina el medio ambiente y viceversa, confirmando su estricta correlación con la elevada incidencia de enteroparasitosis intestinales (Rey, 2001)

De esa manera, se hace necesaria la implantación de medidas básicas de saneamiento e higiene, como medidas profilácticas para la población (OPS, 1996). Así, se hace indispensable para los pacientes con inmunodeficiencias, la implantación de medidas básicas de salud, orientación y asistencia, con el propósito de Que tengan una mejor calidad de vida posible.

En consecuencia, nosotros recomendamos, como mínimo, la instalación de las siguientes medidas preventivas: evitar contacto con el suelo; evitar contacto con animales, inclusive los domésticos; evitar ingerir verduras crudas; hervir siempre el agua para consumo y evitar consumo de alimentos y agua cuya procedencia sea desconocida.

\section{AGRADECIMIENTOS}

Al equipo de técnicos e profesores de la Unidad de Helmintología, del Instituto de Patología Tropical y Salud Pública (IPTSP) de la Universidad Federal de Goiás, en- 
cabezados por la Profa. Julieta L. B. Paço, así como a los alumnos pasantes de graduación y post-graduación, Que de alguna manera contribuyeron con el éxito de este estudio, en especial, LK Vidal, GM Fagundes, RMO Portela y RS Manzi.

\section{REFERENCIAS BIBLIOGRÁFICAS}

Branco, ACIr, Waib, CM \& Oliveira, OCF. 1999. Importância da higiene dos alimentos na epidemiologia das helmintíases: Ocorrência de ovos de helmintos em hortaliças. Revista Brasileira de Análises Clínicas. vol. 31, pp. 3-4.

CETESB- Manual do CETESB. 1989. Helmintos e protozoários patogênicos contagem de ovos e cistos em amostras ambientais. CETESB, São Paulo.

Cimermam, B \& Cimermam, S. 1999. Parasitológia humana. Atheneu, São Paulo.

D’Agosto, M, Ferreira, AFSR, Oliveira, CE, Santos, HH, Maia MC \& Abreu PF. 2000. Contaminação ambiental por formas infectantes de parasitas no bairro de jardim Casablanca, município de luiz de Fora - Minas Gerais. Revista de Patologia Tropical. vol. 29, pp. 101-108.

De Carli,GA. 2000. Parasitologia clinica: Diagnóstico de laboratório dos coccidios e microsporidios intestinais. Cadernos Edipucrs, 14 - Série Farmácia. I, Porto Alegre.

Gassi, TMT, Macedo, LAA, Born, RH, Prota, MG, Cabral, JrD, Santos, AJO, Inglez, PVA \& Homem, ICM. 1984. Classificação ambiental das infeçôes excretadas um exercício de aplicação em fossa séptica. Revista DAE. vol. 44, pp. 277-287.

Gelli, DS, Tachibana, T, Oliveira, IR, Zamboni, CQ, Pacheco, IÁ \& Spiteri, N. 1979. Condições higiênicas - sanitárias de hortaliças comercializadas na cidade de São Paulo ,SP, Brasil. Revista do Instituto Adolfo Lutz. vol. 39, pp. 37-43.

Guilherme, ALF, Araújo, SM, Falavigna, LM, Pupulim, ART, Dias, MLG, Oliveira, HS, Marroco E \& Fukushigue Y. 1999. Prevalência de enteroparasitas em horticultores e hortaliças da feira do produtor de Maringá, Paraná. Revista da Sociedade Brasileira de Medicina Tropical. vol. 32, pp. 405-411.

Ludwig, KM, Frei, F, Álvares, FF \& Ribeiro-Paes, IT. 1999. Correlação entre condições de sanea- mento básico e parasitoses intestinais na população de Assis, Estado de São Paulo. Revista da Sociedade Brasileira de Medicina Tropical. vol. 32, pp. 547:555.

Niehaus, MD, Moore, SR, Patrick, PD, Derr, LD, Lorntz, B, Lima, AA \& Guerrant, RL. 2002. Early childhood diarrhea is associated with diminished cognitive function 4 to 7 years in children in a northeast Brazilian shantytown. American Journal of Tropical Medicine and Hygiene. vol. 66, pp. 590-598.

Oliveira, CAF \& Germano, PML. 1992. Estudo da ocorrência de enteroparasitos em hortaliças comercializadas na região metropolitana de São Paulo, SP, Brasil. I- Pesquisa de helmintos. Revista de Saúde Pública. vol. 26, pp. 283-289.

Oliveira, CAF \& Germano, PML. 1992. Estudo da ocorrência de enteroparasitas em hortaliças comercializadas na região metropolitana de São Paulo, SP, Brasil. 2- Pesquisa de protozoários. Revista de Saúde Pública. vol. 26, pp. 332-335.

Organización Sanitaria Panamericana, Organización Mundial de la Salud, Centros para el Control y Prevención de Enfermedades, Programa Conjunto de las Naciones Unidas sobre el VIH/SIDA(ONUSIDA). 1996. Pautas para la prevención de infecciones oportunistas en personas com $\mathrm{VIH} \mathrm{o}$ SIDA en América Latina y el Caribe. OPS, Washington DC.

Pessoa, SB \& Martins, AV. 1988. Parasitologia médica. Guanabara Koogan, Iled, Rio de laneiro.

Rey, L. 200 I. Parasitologia. Guanabara Koogan, Rio de Janeiro.

Silva, IP, Marzochi, MCA, Coura, LC, Messias, AA \& Marques, S. 1995. Estudo da contaminação por enteroparasitos comercializadas nos supermercados da cidade do Rio de laneiro. Revista da Sociedade Brasileira de Medicina Tropical. vol. 28, pp. 237-241.

Takayanagui, OM, Febronio, LHP, Bergamini, AM, Okino, MHT,Castro e Silva AAMC, Santiago, R, Capuano, DM \& Oliveira, MA. 2000. Fiscalização de hortas produtoras de verduras do Municipio de Ribeirao Preto, SP. Revista da Sociedade Brasileira de Medicina Tropical. vol. 33, pp. 169-174. 
Autor para correspondencia / Author to correspondence:

Marco Túlio Antonio García-Zapata

Instituto de Patologia Tropical e Saúde Pública (IPTSP) / Universidade Federal de Goiás, Goiânia - GO, Brasil, Caixa Postal 12911 - Setor Leste Vila

Nova, 74643-970, Goiania-GO - Brasil

Correo electrónico/ E-mail:

zapata@iptsp.ufg.br

mctulian@yahoo.com.br

Telefax: (62)-3521-1839. 